\title{
Common origin of plasmid encoded alpha- hemolysin genes in Escherichia coli
}

Ylanna Burgos ${ }^{\dagger}$, Lothar Beutin $^{*+}$

\begin{abstract}
Background: Alpha $(\alpha)$-hemolysin is a pore forming cytolysin and serves as a virulence factor in intestinal and extraintestinal pathogenic strains of $E$. coli. It was suggested that the genes encoding $\alpha$-hemolysin (hlyCABD) which can be found on the chromosome and plasmid, were acquired through horizontal gene transfer. Plasmid-encoded $\alpha$-hly is associated with certain enterotoxigenic (ETEC), shigatoxigenic (STEC) and enteropathogenic E. coli (EPEC) strains. In uropathogenic E. coli (UPEC), the $\alpha$-hly genes are located on chromosomal pathogenicity islands. Previous work suggested that plasmid and chromosomally encoded $\alpha$-hly may have evolved independently. This was explored in our study.

Results: We have investigated $11 \alpha$-hly plasmids from animal and human ETEC, STEC and EPEC strains. The size of $\alpha$-hly plasmids ranges from $48-157 \mathrm{~kb}$ and eight plasmids are conjugative. The regulatory gene ( $h l y R$ ) located upstream of the hlyCABD gene operon and an IS911 element located downstream of hlyD are conserved. Chromosomally-encoded $\alpha$-hly operons lack the hlyR and IS911 elements. The DNA sequence of hlyC and hlyA divided the plasmid- and chromosomally-encoded $\alpha$-hemolysins into two clusters. The plasmid-encoded $\alpha$-hly genes could be further divided into three groups based on the insertion of IS1 and IS2 in the regulatory region upstream of the $\alpha$-hly operon. Transcription of the hlyA gene was higher than the housekeeping icdA gene in all strains (rq 4.8 to 143.2). Nucleotide sequence analysis of a chromosomally located $\alpha$-hly determinant in Enterobacter cloacae strain indicates that it originates from an E. coli $\alpha$-hly plasmid.

Conclusion: Our data indicate that plasmids encoding $\alpha$-hly in E. coli descended from a common ancestor independent of the plasmid size and the origin of the strains. Conjugative plasmids could contribute to the spread of the $\alpha$-hly determinant to Enterobacter cloacae. The presence of IS-elements flanking the plasmid-encoded $\alpha$-hly indicate that they might be mobile genetic elements.
\end{abstract}

\section{Background}

Two major types of calcium dependent, pore forming cytolysins of the repeats in toxin (RTX)-family, called alpha- $(\alpha)$ and EHEC-hemolysin (enterohemolysin) were described in strains of Escherichia coli [1,2]. Both types of hemolysins are encoded by polycistronic operons consisting of four genes arranged in the order of hly$C A B D[3,4]$. The product of the $h l y C$ gene is involved in activation of the hemolytic toxin the product of the $h l y A$ gene. The gene products of $h l y B$ and $h l y D$ together with TolC are involved in secretion of the hemolysin through the bacterial cell wall [5].

\footnotetext{
* Correspondence: lothar.beutin@bfr.bund.de

+ Contributed equally

National Reference Laboratory for E. coli, Federal Institute for Risk Assessment (BfR), D12277 Berlin, Germany
}

EHEC-hemolysin is encoded on non-conjugative plasmids in strains of enterohemorrhagic E. coli (EHEC) that cause hemorrhagic diseases in humans $[6,7]$. In contrast, $\alpha$-hemolysin is frequently associated with human uropathogenic E. coli (UPEC) strains [8] and with enterotoxigenic (ETEC), shigatoxigenic (STEC) and enteropathogenic E. coli (EPEC) strains that cause diarrhea and edema disease in animals [9-12].

In UPEC the $\alpha$-hly genes are found on large chromosomal pathogenicity islands (PAI) $[13,14]$. The UPEC O4 (J96) and O6 (536) strains carry each two $\alpha$-hly operons located on different PAIs $[15,16]$, which contain divers junctions and adjacent sequences. This suggests that these loci have evolved independently $[16,17]$. Genetic analysis of chromosomal $\alpha$-hly operons revealed differences in $5^{\prime}$ flanking sequences and toxin expression [18-20].

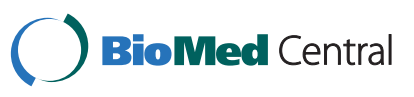


Plasmid-encoded $\alpha$-hly genes were found associated with EPEC O26 strains [21], as well as with ETEC and Shiga toxin 2e (Stx2e) producing STEC strains [9,10,22]. $\alpha$-hly plasmids of $E$. coli were found to differ widely in size, incompatibility groups and conjugational transfer ability $[10,20,21,23]$. So far, only two plasmid $\alpha$-hly operons were completely sequenced. The first is located on the $48 \mathrm{~kb}$ non-conjugative plasmid pHly152 from a murine E. coli strain [24]. The other is located on the $157 \mathrm{~kb}$ conjugative plasmid pEO5 of a human EPEC O26 strain [21]. Interestingly, despite the differences between pHly152 and pEO5, the DNA sequence of their $\alpha$-hly operons are $99.2 \%$ similar while the sequence of the upstream regulatory $h l y R$ region is $98.8 \%$ similar [21]. Importantly, the plasmid-inherited $\alpha$-hly are less similar (96.0-96.4\%) to the chromosomally inherited $\alpha-h l y C A B D$ located on PAI I [GenBank AJ488511] and PAI II [GenBank AJ494981] of the E. coli strain 536 $[18,21]$. Moreover, chromosomally and plasmid-inherited $\alpha$ - $h l y$ operons also differ also for their 5 regulatory $h l y R$ region. These findings suggest that the plasmid and chromosomal $\alpha$-hly operons have evolved in parallel.

Studies on hemolysins of other bacterial species revealed similarities between the $E$. coli $\alpha$-hemolysin genes and the Enterobacter, Proteus, Morganella and Mannheimia operons [25,26]. Codon usages base composition studies suggested that the $\alpha$-hlyCABD genes of E. coli were originated from Proteus, Morganella or Mannheimia species [25,27]. Transposon-like structures found in the neighborhood of plasmid pHly152 and pEO5 encoded $\alpha$-hly operons suggest that these were acquired by horizontal gene transfer [20,21].

The fact that the $\alpha-h l y C A B D$ genes and their adjacent regions on pHly152 and pEO5 were highly similar to each other prompted us to investigate the genetic relationship between plasmid and chromosomal inherited $\alpha$-hly operons in more strains of E. coli and in Enterobacter cloacae. Our results indicate that plasmid $\alpha$-hly operons are highly similar regardless of differences in the plasmid backbone sequences, bacterial host and their source, suggesting that they have evolved from a common origin.

\section{Results}

\section{Characterization of $\alpha$-hly plasmids in E. coli strains}

Nucleotide sequence analysis of the pEO5 $\alpha-h l y C A B D$ operon and its 5 ' region revealed high similarity to the corresponding DNA segment of the $\alpha$-hly plasmid pHly 152 [21]. Since pEO5 and pHly152 differ in their origin, size and conjugative transfer, we investigated if plasmid $\alpha$-hly operons have a common origin and evolved independently of chromosomal $\alpha$-hlyCABD genes in E. coli.

In order to explore the genetic relationship between plasmid $\alpha$-hly genes we investigated five $\alpha$-hly plasmids originating from canine ETEC strains and four plasmids of porcine ETEC and STEC strains (Table 1). $\alpha$-hemolysin plasmids were detected by DNA-hybridization of Southern blotted plasmid DNA as described in Material and Methods (Fig. 1). The size of $\alpha$-hly plasmids from dogs, pigs, mouse, cattle and human origin varied between $48 \mathrm{~kb}$ to $157 \mathrm{~kb}$ and other than pEO13, pEO14 and pEO860 all other plasmids were found transferable by conjugation (Table 1 ). Plasmid profile analysis has shown that the $\alpha$-hly-plasmids are frequently found together with other large plasmids (Fig. 1).

In addition, we investigated four $E$. coli and an $E$. cloacae strain with chromosomal $\alpha$-hly operons (Table 1 ). A BLAST search using pEO5 [GeneBank FM180012] and phly152 [GeneBank M14107] sequences between $h l y R$ and $h l y C$ and downstream of $h l y D$ revealed no similarity with sequences of chromosomal $\alpha$-hly genes in strains CFT073 [GeneBank AE014075], UTI89 [CP000243] and 536 [CP000247].

\section{Analysis of the plasmid and chromosomal upstream $\alpha$-hly operons}

Based on the pEO5 DNA sequence (Fig. 2) we developed specific primers for amplification of fragments within the $h l y R$, and $h l y R-h l y C$ regions (Table 2 ). In addition, we developed specific PCRs for the upstream $h l y C$ sequences of the chromosomal $\alpha$-hemolysin operons in PAI I and PAI II of strain 536 [15] (Table 2). We performed PCR analysis of all strains carrying plasmid and chromosomal $\alpha$-hly operons; strains carrying $\alpha$-hlyplasmids pEO5 and pHly152 and 536 served as positive controls. The results are summarized in Table 1.

Primers $1 \mathrm{f} / \mathrm{r}$ are specific for the upstream $h l y C$ region in pEO5 and yielded a PCR product of 678 bp (Fig. 2). PCR products of the same size were obtained with all strains carrying $\alpha$-hly plasmids, except 84/S (pEO14); restriction enzyme analysis revealed all the fragments had a similar HinfI profile (data not shown). Primers 1f/ $\mathrm{r}$ gave no products using $E$. coli strains carrying chromosomally encoded $\alpha$ - $h l y$ as template with the exception of the E. cloacae strain KK6-16 which yielded a PCR product; DNA sequencing revealed a 778 bp fragment [GenBank FM210352, position 72-849] (Table 1).

Primers $32 \mathrm{f} / \mathrm{r}$ spanning the region between $h l y R$ and the "phly152" segment amplify a 671 bp product in pEO5 [GenBank FM180012, position 597-1267] (Fig. 2). A PCR product of the same size was obtained with pEO5 and derivative plasmids as well as with plasmids pEO9 [GenBank FM210248 position 427-1097], pEO13 and pEO860 (Table 1, Fig. 3). Primers $32 \mathrm{f} / \mathrm{r}$ yielded PCR products of 2007 bp with pEO11, [GenBank FM210249, position 3922398), pHly152 and pEO12, and 2784 bp PCR products with pEO853 [GenBank FM10347 position 399-3182], pEO855 and pEO857 (Table 1). All amplicons of a given size (671 bp, $2007 \mathrm{bp}$ and $2784 \mathrm{bp}$ ), yielded a similar HinfI 
Table 1 Relevant properties of strains carrying plasmid and chromosomally encoded $\alpha$-hly determinants

\begin{tabular}{|c|c|c|c|c|c|c|c|c|c|c|}
\hline \multirow[b]{2}{*}{ strain } & \multirow[b]{2}{*}{ Serotype $^{b}$} & \multirow[b]{2}{*}{ Origin, reference $^{d}$} & \multirow[b]{2}{*}{ hly-plasmid (kb) } & \multirow[b]{2}{*}{ Plasmid group } & \multicolumn{6}{|c|}{ PCR products with primers pairs ${ }^{a}$} \\
\hline & & & & & $\begin{array}{c}1 \mathrm{f} / \mathrm{r} \\
(678 \mathrm{bp})\end{array}$ & $\begin{array}{c}32 \mathrm{f} / \mathrm{r} \\
(671 \mathrm{bp})\end{array}$ & $\begin{array}{c}44 f / r \\
(685 \mathrm{bp})\end{array}$ & $\begin{array}{c}99 \mathrm{f} / \mathrm{r} \\
(650 \mathrm{bp})\end{array}$ & $\begin{array}{c}72 \mathrm{f} / \mathrm{r} \\
(695 \mathrm{bp})\end{array}$ & $\begin{array}{c}81 \mathrm{f} / \mathrm{r} \\
(773 \mathrm{bp})\end{array}$ \\
\hline$\overline{C 4115}$ & $\mathrm{O} 26:[\mathrm{H} 11]$ & human, EPEC [21] & pEO5 (157) & 1 & + & + & + & + & - & - \\
\hline TPE422 & Or:H48 & $\begin{array}{l}\text { E. coli K12 (pEO5) } \\
\text { [21] }\end{array}$ & pEO5 (157) & 1 & + & + & + & + & - & - \\
\hline CB9866 & $\mathrm{O} 26:[\mathrm{H} 11]$ & cattle, EPEC [21] & pEO5 (157) & 1 & + & + & + & + & - & - \\
\hline CB1027 & $\mathrm{O} 26:[\mathrm{H} 11]$ & human, EPEC [21] & pEO5 (157) & 1 & + & + & + & + & - & - \\
\hline CB1030 & $\mathrm{O} 26:[\mathrm{H} 11]$ & human, EPEC [21] & pEO5 (157) & 1 & + & + & + & + & - & - \\
\hline IP187 & $\mathrm{O} 26:[\mathrm{H} 11]$ & human, EPEC [21] & pEO5 (157) & 1 & + & + & + & + & - & - \\
\hline $84 / 2195$ & Ont:H10 & $\operatorname{dog}[10]$ & pEO9 (146) & 1 & + & + & + & + & - & - \\
\hline $84-R$ & $\mathrm{O} 121: \mathrm{H} 46$ & $\operatorname{dog}[10]$ & pEO13 (97) & 1 & + & + & + & + & - & - \\
\hline 374 & Or:H48 & mouse [24] & pHly152 (48) & 2 & + & e) & + & + & - & - \\
\hline $84-3208$ & $\mathrm{O} 42: \mathrm{H} 37$ & dog, ETEC[10] & pEO11 (48) & 2 & + & e) & + & + & - & - \\
\hline $84-2573$ & O70:NM & dog, ETEC [10] & pEO12 (48) & 2 & + & e) & + & + & - & - \\
\hline CB853 & O138:H14 & pig, STEC [29] & pEO853 (145) & 3 & + & f) & g) & + & - & - \\
\hline CB855 & O138:NM & pig, STEC [29] & pEO855 (140) & 3 & + & f) & g) & + & - & - \\
\hline CB857 & O157:NM & pig, ETEC [42] & pEO857 (97) & 3 & + & f) & g) & + & - & - \\
\hline CB860 & O149:H10 & pig, ETEC [42] & pEO860 (48) & single & + & + & g) & + & - & - \\
\hline $84-25$ & $\mathrm{O} 75: \mathrm{H} 2$ & $\operatorname{dog}[10]$ & pEO14 (97) & single & - & - & - & - & - & - \\
\hline $536^{h}$ & O6:K15:H31 & human UPEC [20] & - & n.a & - & - & - & - & + & + \\
\hline $536-14$ & O6:K15:H31 & $\begin{array}{l}\text { PAl I deletion } \\
\text { mutant of } 536 \text { [20] }\end{array}$ & - & n.a & - & - & - & - & + & - \\
\hline $695 / 83$ & O126:H27 & human [19] & - & n.a & - & - & - & - & - & i) \\
\hline$J 96^{h}$ & $\mathrm{O} 4: \mathrm{K} 6$ & human UPEC [46] & - & n.a & - & - & - & - & + & j) \\
\hline KK6-16 & E. cloacae & human [26] & - & n.a & k) & - & - & - & - & - \\
\hline
\end{tabular}

a) primer pairs and size of the PCR products obtained with strains TPE422 (pEO5) (primers 1f/r, 32f/r and 44f/r) and 536 (primers $81 f / r$ and $72 f / r$ ) (see Table 2).

$+=$ a PCR product of the same size as obtained with strains TPE422 (pEO5) or 536, respectively.

- = no PCR product obtained

$\mathrm{PCR}$ products with other sizes than obtained with the reference strains are indicated for their length in bp.

b) O:H serotype, Or = rough LPS, Ont = O-antigen not typable, NM = non motile

c) $\alpha=$ alpha-hemolytic ( $\alpha$-hly genes), e-hly = enterohemolytic (EHEC-hly genes)

d) All strains were from feces if not otherwise indicated, ETEC = enterotoxigenic E. coli, STEC = shigatoxigenic E. coli, UPEC = uropathogenic E. Coli

e) $2000 \mathrm{bp}$ PCR product (2007 bp as calculated from the nucleotide sequence of pEO11 [GenBank FM210249]

f) $2900 \mathrm{bp} \mathrm{PCR}$ product (2868 bp as calculated from the nucleotide sequence of pEO860) [GenBank FM210351]

g) $1500 \mathrm{bp}$ PCR products

h) strain carries two $\alpha$-hly determinants in the chromosome

i) 950 bp PCR product

j) $860 \mathrm{bp} P C R$ product

k) $778 \mathrm{bp} \mathrm{PCR} \mathrm{product} \mathrm{(calculated} \mathrm{from} \mathrm{nucleotide} \mathrm{sequencing} \mathrm{of} \mathrm{KK6-16} \mathrm{[GenBank} \mathrm{FM210352])}$

restriction pattern (data not shown). Strains with chromosomally encoded $\alpha$-hemolysin gave no products in the $32 \mathrm{f} / \mathrm{r}$ PCR, as well as strain 84/2 S carrying plasmid pEO14 (Table 1).

Primer pair $44 \mathrm{f} / \mathrm{r}$ (Fig. 2) is specific for $h l y R$ and amplified related sequences in all $E$. coli carrying $\alpha$-hly plasmids except pEO14. The $h l y R$ PCR product was 685 bp (pEO5) [GeneBank FM180012, position 167-851] for all plasmids except for pEO853, pEO855, pEO857 and pEO860 which generated amplicons of about $1400 \mathrm{bp}$ (Table 1). The $685 \mathrm{bp}$ and $1400 \mathrm{bp}$ size PCR products yielded similar HinfI restriction profiles, respectively.
Strains with chromosomally inherited $\alpha$-hly genes were negative for $h l y R$ sequences (Table 1 ).

None of the strains with $\alpha$-hly plasmids, or the E. cloacae strain KK6-16 yielded PCR products with primer pairs $81 \mathrm{f} / \mathrm{r}$ and $72 \mathrm{f} / \mathrm{r}$, that are specific for PAI I and PAI II $\alpha$-hemolysins (Table 2) [17]. All strains with chromosomal $\alpha$-hly except KK6-16 produced PCR products with one or both of these primer pairs (Table 1). Taken together, the PCR typing indicated that all plasmid $\alpha$-hly except pEO14 were similar for the regulatory regions located upstream of the $h l y$-genes which differed from the chromosomal $\alpha$-hly operons. 


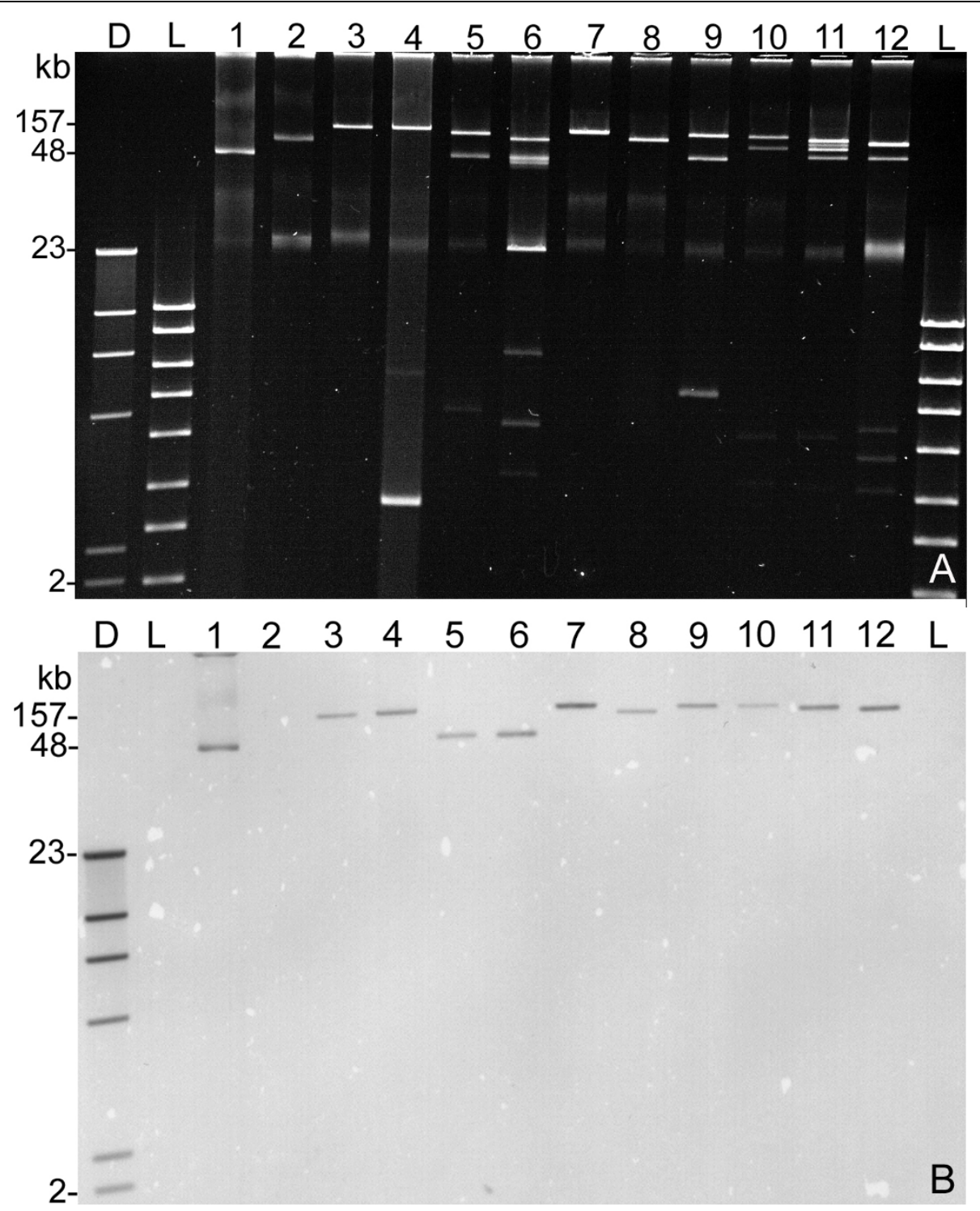

Figure 1 Detection of plasmid encoded $\boldsymbol{\alpha}$-hly genes in $\boldsymbol{E}$. coli strains. A) Agarose gel (0.7\%) with plasmid preparations obtained from E. coli strains. Lanes: $\mathrm{D}=$ digoxigenin-labelled molecular weight standard II (Roche); $\mathrm{L}=$ molecular weight standard hyperladder I (Bioline); $1=374$ (phly152); 2 = TPE1313 (pO157); 3 = TPE422 (pEO5); 4 = TPE1030 (pEO5); 5 = 84-3208 (pEO11); 6 = 84-2573 (pEO12); $7=84-2195$ (pEO9); 8 = 84-2 S (pEO14); 9 = 84-R (pEO13); $10=$ CB853 (pEO853); 11 = CB855 (pEO855); 12 = CB857 (pEO857). B) Southern hybridization patterns of plasmid DNA from lanes 1-12 with the $\alpha$-hlyA specific digoxigenin labelled gene probe generated with primers $10 \mathrm{f} / \mathrm{r}$ from plasmid pEO5 DNA. The size of hybridizing $\alpha$-hly plasmids varies from 48 (lane 1) to $157 \mathrm{~kb}$ (lane 3).

\section{Genetic analysis of the region between $h l y R$ and $h l y C$ in} $\alpha$-hly plasmids

A 464 bp DNA segment that carries a promoter $\left(\mathrm{pHhly}_{\mathrm{L}}\right.$ ) for expression of $\alpha$-hly-genes is located directly upstream of the $h l y C$ gene in plasmid pHly152 [24] [GenBank M14107]. A 466 bp region with $98.9 \%$ sequence homology was found upstream of $h l y C$ in pEO5 [21]. The "phly152" region is not present in E. coli strains containing chromosomal $\alpha$-hly genes [20] (this work). Sequences highly homologous to a large part of the "phly152" region were found in all $\alpha$-hly plasmids investigated here, except pEO14. Comparison of the complete $466 \mathrm{bp}$ "phly152" DNA stretch of plasmids pEO5 [GenBank FM180012], pEO9 [FM210248], pEO853 [FM210347], pEO11 [FM210249] and pEO860 [FM210351] revealed similarities from $97.9 \%$ to $100 \%$. Interestingly, a 427 bp fragment with $93 \%$ similarity to the "phly152" segment was found upstream of $h l y C$ in the E. cloacae strain KK6-16 [GenBank FM210352, position 1-427].

Sequences specific for $h l y R$ [GenBank X07565], a regulatory region located about $2000 \mathrm{bp}$ upstream of the 


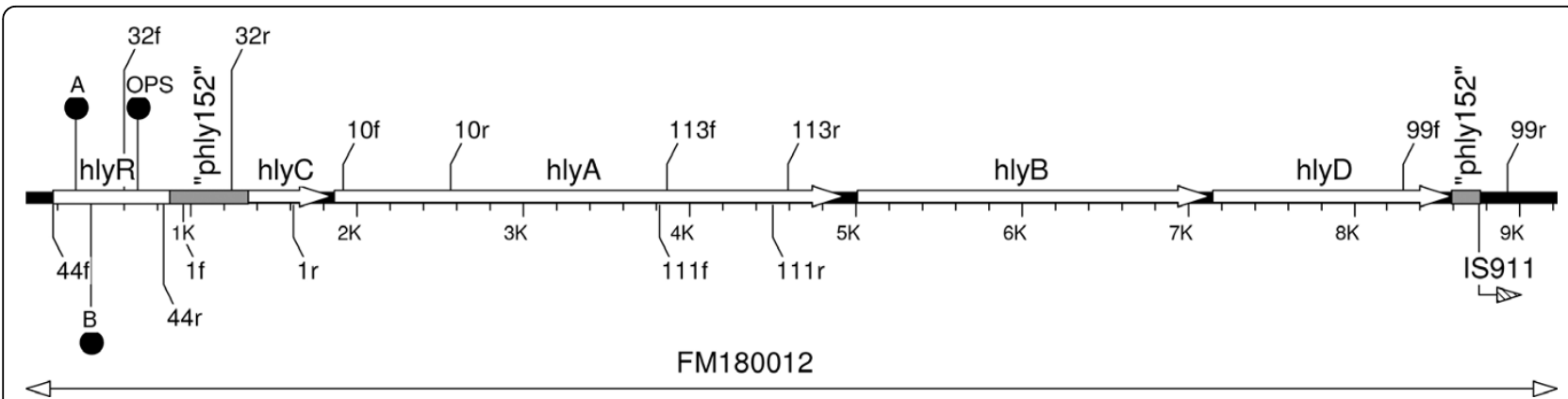

Figure 2 Map of the $\boldsymbol{\alpha}$-hly region of plasmid pEO5 (FM180012). The positions of PCR-primers used for investigation of strains with plasmid and chromosomally inherited $\alpha$-hly genes are indicated as leaders carrying the primer designations (Table 2). Regulatory sequences inside the hlyR gene (A, B and OPS) are shown as filled ballons. "phly152" is a stretch of non-coding DNA showing strong homology to corresponding regions in the $\alpha$-hly plasmid pHly152.

$\alpha$-hly determinant in pHly152 [28] were present in all $\alpha$-hly plasmids except pEO14. The hlyR regions of five representative plasmids (pHly152, pEO5, pEO9, pEO11 and $\mathrm{pEO} 853$ ) were analyzed and compared to each other (Fig. 3). Short DNA sequences that were reported to be involved in regulation of $\alpha$-hly expression located inside $h l y R$, i.e regulatory sequences A and B [28] and the "operon polarity suppressor (ops) [18], were identified in the corresponding $h l y R$ region of the five plasmids. A clustal analysis performed with a $565 \mathrm{bp}$ segment of the $h l y R$ region beginning with the regulatory sequence $\mathrm{A}$ to the end of the $h l y R$ region revealed 98.8 to $100 \%$ similarity between these five plasmids.

\section{IS1 and IS2 elements are present in the $\alpha$ hly-upstream} region of plasmid hly

According to the length of PCR products obtained for the region located between $h l y R$ and $h l y C$ genes three groups of $\alpha$-hly plasmids were established (Table 1). "Group 1" is represented by pEO5, its homologues from other E. coli $\mathrm{O} 26$ strains and by pEO9 and pEO13. "Group 2" is represented by pHly152, pEO11 and pEO12. "Group 3" is formed by plasmids pEO853, pEO855 and pEO857 from porcine strains. Two strains with $\alpha$-hly plasmids pEO14 and pEO860 showed individual patterns by PCR-typing (Table 1 ). In order to explore the differences between the major groups of $\alpha$ $h l y$-plasmids we determined the nucleotide sequence of the region located between $h l y R$ and $h l y C$ of three representative plasmids, namely pEO9 [GenBank FM210248], pEO11 [FM210249] and pEO853 [FM210347] (Fig. 3).

Major differences between the $\alpha$-hly plasmids in the region between $h l y R$ and $h l y C$ caused by insertion of IS1 and IS2. While "group 1" plasmids (pEO5, pEO9 and pEO13) carry no IS elements all "group 2" plasmids

Table 2 Specific PCRs for identification of plasmid and chromosomally inherited $\alpha$-hly determinants

\begin{tabular}{|c|c|c|c|c|c|}
\hline DNA-target (position in sequence) & GenBank Accession & Primer & nucleotide sequence $\left(5^{\prime}-3^{\prime}\right)$ & $\operatorname{Tm}\left({ }^{\circ} \mathrm{C}\right)$ & PCR product bp \\
\hline hlyA (1915-1936) (2560-2580) & FM180012 & $\begin{array}{l}10 f \\
10 r\end{array}$ & $\begin{array}{l}\text { GCTGCAAATAAATTGCACTCAG } \\
\text { CCCTGCACCGATATTATCAAG }\end{array}$ & 53.1 & 666 \\
\hline "pHly152" (953-974) \&hlyC (1612-1630) & FM180012 & $\begin{array}{l}\text { If } \\
\text { 1r }\end{array}$ & $\begin{array}{l}\text { GTAGTTCAAAAGACAACTCGTG } \\
\text { ATCCCCGAAAGGAGCAATC }\end{array}$ & 50.6 & 678 \\
\hline hlyR (597-618) \& "pHly152" (1246-1267) & FM180012 & $\begin{array}{l}32 f \\
32 r\end{array}$ & $\begin{array}{l}\text { GTCTTGCCGTACAATAATTTCC } \\
\text { TCCGTTIAATGTCATAACTCGC }\end{array}$ & 56.5 & $671^{a}$ \\
\hline hlyR (167-188) (830-851) & FM180012 & $\begin{array}{l}44 f \\
44\end{array}$ & $\begin{array}{l}\text { ATTCCAAGCGAAGTCCATCCCC } \\
\text { CATAAAGCATGATGCCACCACG }\end{array}$ & 66.5 & $685^{\mathrm{a}}$ \\
\hline hlyA (3817-3839) (4497-4518) & FM180012 & $\begin{array}{l}111 f \\
111 r\end{array}$ & $\begin{array}{l}\text { GATGGCACAAAAGCAACCGAAG } \\
\text { TTCTCGCTTGAAGGCCACATCC }\end{array}$ & 55.4 & 702 \\
\hline hlyA (3865-3883) (4592-4613) & FM180012 & $\begin{array}{l}113 f \\
113 r\end{array}$ & $\begin{array}{l}\text { CTTGGTGGCGATGTTAAGG } \\
\text { GACTCTIITICAAACCAGTTCC }\end{array}$ & 53.5 & 749 \\
\hline hlyD (8297-8319) \& IS911 (8925-8946) & FM180012 & $\begin{array}{l}99 f \\
99 r\end{array}$ & $\begin{array}{l}\text { GCAGAATGCCATCATTAAAGTG } \\
\text { CCATGTAGCTCAAGTATCTGAC }\end{array}$ & 53.8 & 650 \\
\hline PAl I (536) (44506-44524) \&hlyC (45278-45299) & AJ488511 & $\begin{array}{l}81 f \\
81 r\end{array}$ & $\begin{array}{l}\text { CCTGTGACACTTCTCTTGC } \\
\text { CCCAAGAACCTCTAATGGATTG }\end{array}$ & 52.3 & $773^{\mathrm{a}}$ \\
\hline PAI II (536) (31974-31995) \&hlyC (32650-32668) & AJ494981 & $\begin{array}{l}72 f \\
72 r\end{array}$ & $\begin{array}{l}\text { CCCAACTACAATATGCAACAGG } \\
\text { CGCCAATAGAGTTGCCTC }\end{array}$ & 51.9 & 695 \\
\hline
\end{tabular}

a) PCR products of different lengths were obtained with these primers depending on the DNA template (see Table 1) 


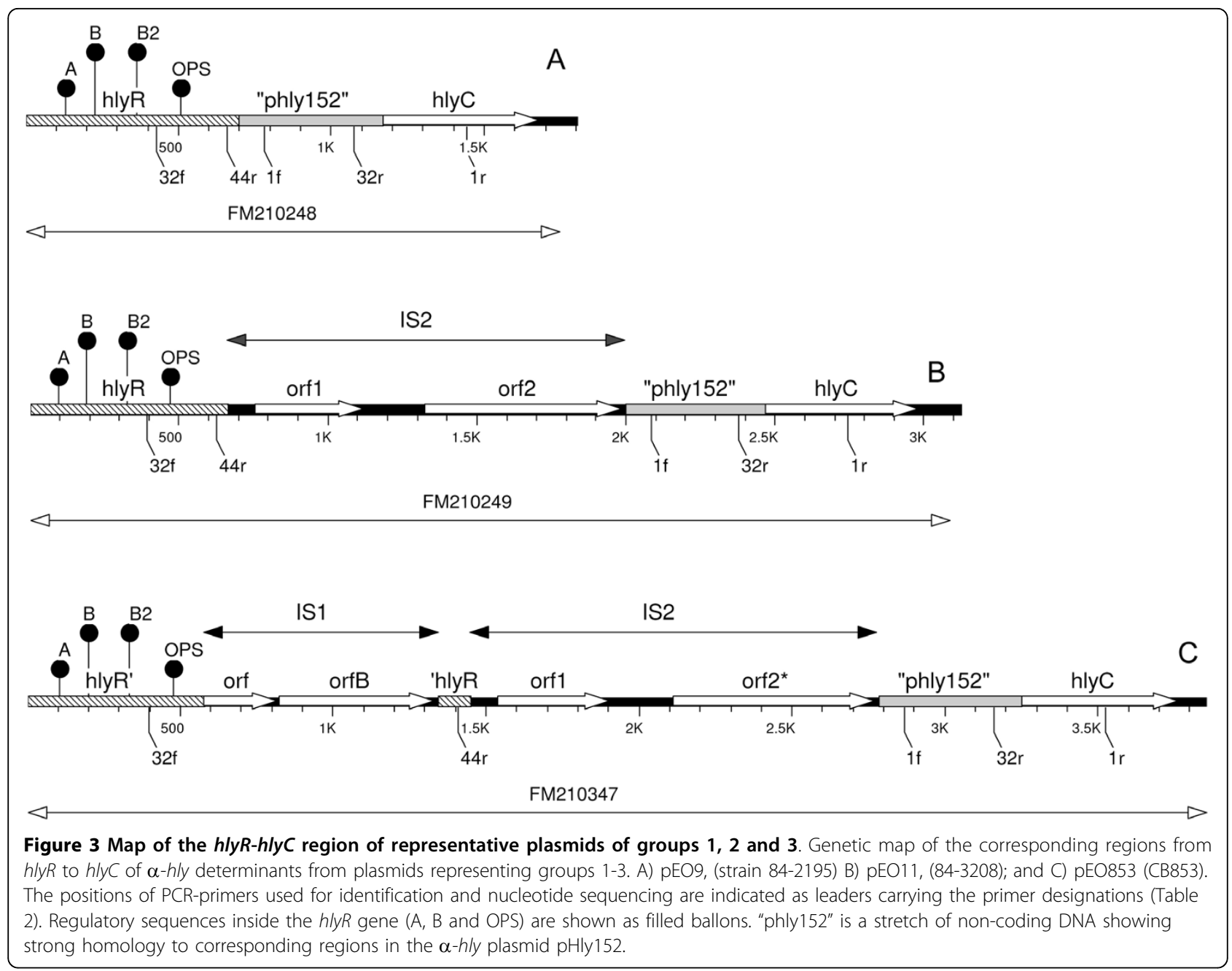

(phly152, pEO11 and pEO12) carry an IS2 element inserted directly downstream of the 3 ' end of $h l y R$ (5' CCTGG 3') in pEO11. A 326 bp part of the IS2 element was previously described in pHly152 [GenBank M14107] [24], it is $99.4 \%$ identical to the corresponding IS2 element of pEO11. The IS2 elements in pEO11 and pHly152 are inserted at the DNA same site and are both flanked by the duplicated 5' CCTGG 3' DNA sequence.

Plasmids belonging to "group 3", which were all from pig strains (pEO853, pEO855 and pEO857), carry two IS elements in the region between $h l y R$ to $h l y C$. In pEO853, the 786 bp IS1 is inserted immediately downstream of the hlyR internal sequence 5 AACAAAATT 3'. This 9 bp DNA stretch is repeated at the right hand end of the inserted IS1 and followed by the $94 \mathrm{bp}$ residual 3 ' end of the $h l y R$ region (Fig. 3). The IS2 element of pEO853 is $99.8 \%$ similar to that of pEO11 and inserted at the same position as in "group 2" plasmids pEO11 and pHly152.

\section{Investigation of $h l y R$-hlyC region of STEC strains of} porcine origin

We used the primers specific for the region between $h l y R$ to $h l y C$ (Table 1 ) to investigate $26 \alpha$-hemolysin/ stx2e STEC strains from diseased pigs or pork meat [29]. PCR products were obtained from all. According to the length of the amplicons generated with primers $1 \mathrm{f} / \mathrm{r}, 32 \mathrm{f} / \mathrm{r}$ and $44 \mathrm{f} / \mathrm{r}$ all but one strain showed patterns indicating the presence of a "group 2" or "group 3" plasmid with IS-elements in the region between $h l y R$ and $h l y C$ (Table 3 ). The PCR-profiles were closely associated with serotypes of strains causing edema disease in pigs (O138:H14, O139:H1 and O141:H4) suggesting that $\alpha$-hly plasmids are conserved in these strains.

\section{Sequence analysis of the $h l y C$ gene of plasmid and chromosomal $\alpha$-hemolysin}

The fact that $\alpha-h l y$ plasmids were similar for the regulatory sequences upstream of the $\alpha$-hly operon prompted us to analyze the coding sequence of seven plasmid 
Table 3 Detection of $\alpha$-hly plasmid specific sequences in porcine STEC strains

\begin{tabular}{ccccccc}
\hline \multicolumn{7}{c}{ Size of PCR products with primers } \\
\hline Serotype & No. strains & Plasmid group & $\mathbf{1 f + 1 r}$ & $\mathbf{3 2 f}+\mathbf{3 2 r}$ & $\mathbf{4 4} \mathbf{f}+\mathbf{4 4 r}$ & $\mathbf{9 9} \mathbf{f}+\mathbf{9 9 r}$ \\
\hline $\mathrm{O} 138: \mathrm{H} 14^{\mathrm{b}}$ & 4 & 3 & 678 & 2900 & 1500 & 650 \\
$\mathrm{O} 139: \mathrm{H} 1^{\mathrm{b}}$ & 9 & 3 & 678 & 2900 & 1500 & 650 \\
$\mathrm{O} 139: \mathrm{H} 1^{\mathrm{b}}$ & 1 & single & 678 & 2000 & 1500 & 650 \\
$\mathrm{O} 141: \mathrm{H}^{\mathrm{b}}$ & 7 & 3 & 678 & 2900 & 1500 & 650 \\
$\mathrm{O} 141: \mathrm{H}^{\mathrm{b}}$ & 3 & 2 & 678 & 2000 & 685 & 650 \\
$\mathrm{O} 2: \mathrm{H} 32^{\mathrm{c}}$ & 1 & 2 & 678 & 2000 & 685 & 650 \\
$\mathrm{O} 36: \mathrm{H} 19^{\mathrm{c}}$ & 1 & 2 & 678 & 2000 & 685 & 650 \\
\hline
\end{tabular}

a) size of the PCR products (bp) with primers $1 \mathrm{f} / \mathrm{r}, 32 \mathrm{f} / \mathrm{r}, 44 \mathrm{f} / \mathrm{r}$ and $99 \mathrm{f} / \mathrm{r}$ as described in Tables $1+2$. Digestion of PCR products by Hinfl resulted in identical patterns between strains corresponding to the size of the PCR products obtained (Table 1)

b) Strains isolated from feces of diseased pigs [29]

c) Strains isolated from pork meat [29]

hlyC genes, namely pEO9 [GenBank FM210248], pEO860 [FM210351], pEO13 [FM210348], pEO14 [FM210350], pEO11 [FM210249], pEO853 [FM210347], and pEO12 [FM210349] (Table 1). We used Clustal W analysis to compare the DNA sequences of the plasmid $h l y C$ genes and the chromosomal $h l y C$ genes of strain 536, PAI [GenBank AJ488511] and PAI II [AJ494981] UTI98 [CP000243], CFT073 [AE014075], J96 [M14107] and that of the E. cloacae strain KK6-16 [FM210352]. All plasmid $h l y C$ sequences, except that of pEO14, showed 99.2 to $100 \%$ nucleotide sequence homology to each other and were grouped into one cluster (Fig. 4). A second cluster ( $98.5 \%$ to $99.6 \%$ similarity) was formed by the chromosomal and pEO14 hlyC genes (Fig. 4). The $h l y C$ gene encoded by pEO14 was most similar to that of PAI II from strain 536 (99.2\% homology). The $h l y \mathrm{C}$ genes of all other $\alpha$-hly plasmids showed 94.995.9\% homology to chromosomal $h l y C$ genes of E. coli. The amino acid (aa) sequences of $h l y C$ translation products revealed five aa-exchanges (positions 3, 5, 40, 51, and 160) in the 170 aa-sequence that were closely associated with the origin (plasmid or chromosome) of the E. coli $h l y C$ genes (data not shown).

The $h l y C$ gene of the E. cloacae strain KK6-16 was more distant for its nucleotide and aa sequence from both the E. coli plasmid and chromosomal hlyC gene clusters and most similar to chromosomal PAI I, PAI II (98.2\%) and pEO13 (97.2\%) hlyC genes (Fig. 4).

\section{Comparison of nucleotide sequences of plasmid and chromosomal $\alpha-h / y A$ genes}

Comparing the nucleotide sequences of hlyA revealed significant differences between chromosomal and plasmid genes. By BLAST search, a 755 bp stretch of the hlyA gene (position 3000-4095 in the pHly152 sequence, GenBank M14105) was found only $91 \%$ similar to the corresponding regions of the chromosomal hlyA genes from PAI I [AJ488511] and PAI II [AJ494981].
In order to explore the differences between plasmid and chromosomal hlyA genes we have developed PCR primers (111f/r and 113f/r from GenBank FM180012, Table 2) for amplification of this DNA region. The nucleotide sequence of the corresponding $633 \mathrm{bp}$ PCR products from strains with $\alpha$-hly plasmids and from E. cloacae strain KK6-16 was determined. The results are presented in Fig. 5. Except for pEO14, all plasmid encoded $h l y A$ internal sequences were very similar to each other with a maximum difference of $1.4 \%$ (pHly152 and pEO13). In contrast, chromosomal hlyA genes showed differences of up to $9.5 \%$ when compared to each other (J96 compared to 536 both PAI I and PAI II). The 211 aa HlyA translation products showed aa-exchanges at positions 58 and 78 that were associated with the E. coli plasmid or chromosomal origin of the genes (data not shown).

The nucleotide sequence of the $h l y A$ region on plasmid pEO14 was found closely related to the chromosomal hlyA gene of strain UTI98 ( $0.6 \%$ difference), and showed $5-6 \%$ sequence differences to all other $\alpha$-hly-plasmids. Interestingly, the $E$. cloacae $h l y A$ gene sequence was found $99 \%$ similar to that of plasmids pEO5 and pEO9 and more distantly related to the E. coli chromosomal $h l y A$ genes (2.6 to $10.4 \%$ differences).

\section{IS911 is present downstream of $h l y D$ in strains carrying $\alpha$-hly plasmids}

It was suggested that the $h l y C A B D$ operons were spread in E. coli by mobile genetic elements [20] and a truncated IS911 segment of 254 bp was found located closely and downstream of the $h l y D$ gene in plasmid pEO5 [21]. In order to investigate the other $\alpha$-hly plasmids for the presence of this element we developed PCR-primers (99f/r) encompassing a 650 bp stretch of DNA starting inside $h l y D$ and ending inside the IS911 sequence. All $\alpha$-hly plasmids except pEO14 yielded a PCR product. None of the strains carrying chromosomal $\alpha$-hly genes 

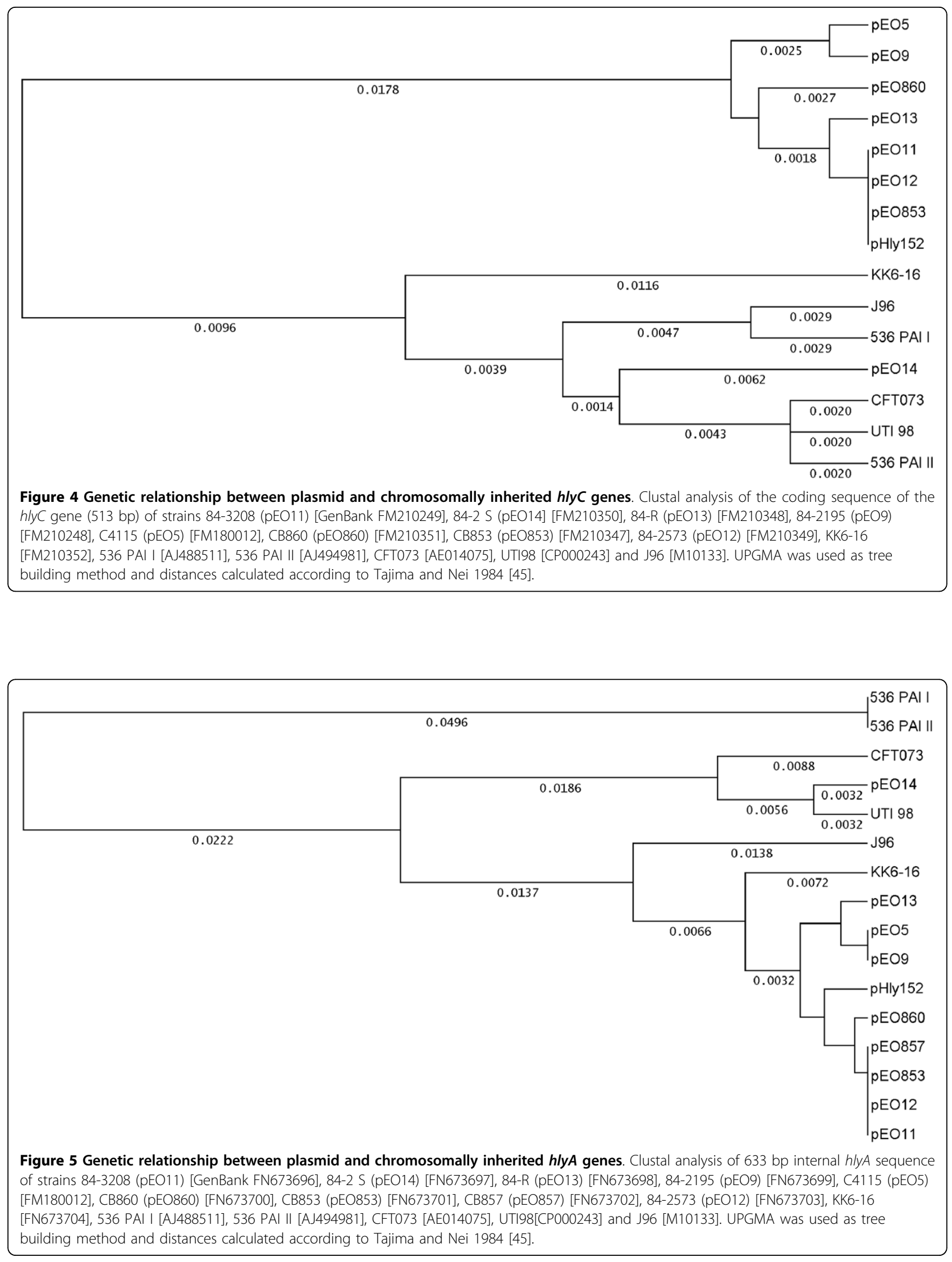
reacted positive with this PCR (Table 1). The nucleotide sequence of the $579 \mathrm{bp}$ amplicons from nine $\alpha$-hly plasmids (strains CB860 [GenBank FN678780], CB857 [FN678781], CB853 [FN678782], 84-3208 [FN678783], 84-2573 [FN678784], 374 [FN678785], 84-R [FN678786], 84-2195 [FN678787] and CB855 [FN678788] were compared by Clustal $\mathrm{W}$ analysis. The sequences were 99.5-100\% identical, indicating that the 3 ' part of the $h l y D$ gene and downstream IS911 sequences are conserved among plasmid $\alpha$-hly determinants. In addition, all 26 STEC strains from pigs or pork meat that carried $\alpha$-hly-plasmids (Table 3 ) yielded $650 \mathrm{bp}$ products with primers $99 \mathrm{f} / \mathrm{r}$, that showed similar HinfI digestion profiles $(257,222$ and $171 \mathrm{bp})$ to those of the sequenced plasmids [FN678782-88] indicating that the hlyD-IS911 region is conserved in these strains.

\section{Transcriptional analysis of plasmid and chromosomal $\alpha$-hlyA genes}

We investigated if the presence of IS elements in the regulatory region upstream $h l y C$ has an affect on transcription of the $\alpha-h l y A$ gene. Phenotypically, all strains with $\alpha$-hly plasmids showed large and clear zones of hemolysis on blood agar plates similar to that found with strains carrying chromosomally inherited $\alpha$-hly genes. An exception was made for strains 536-14 (the PAI I deletion mutant of strain 536) and the wildtype strain 695/83 (Table 1), which generated small, turbid zones of hemolysis on blood agar plates [19].

We compared the transcriptional activity of $15 \mathrm{E}$. coli strains carrying plasmid and chromosomal $\alpha$-hly operons by analyzing the mRNA transcription level of the $\alpha-h l y A$ gene in a relative quantification (rq) assay by Real-Time PCR. The E. coli icdA housekeeping gene was used as a standard (Fig. 6). Transcription of the $h l y A$ gene was higher than $i c d A$ in all strains (rq 4.8 to 143.2). Relatively low hlyA transcription rates (rq 4.8 and 9.7) were found with poor hemolysin producing strains 536-14 and 695/83. Strains carrying "group 1" $\alpha$-hly plasmids (pEO5, pEO9 and pEO13) as well as pEO14 showed significantly (95\% confidence intervals) lower transcription rates ( $\mathrm{rq} 14.4$-24.3) compared to "group 2" and "group 3" strains with IS elements inserted upstream $h l y C$ (rq 56.7 to 143.2). Significant differences in $h l y A$ transcription rates were found between individual strains carrying "group 2" and "group 3" plasmids but they could not be clearly assigned to one of two groups. Except for pEO12 and pEO853, all "group 2" and "group 3" strains showed hlyA transcription rates that were not significantly different from those of strains 536 and J96, the latter carry each two chromosomally inherited $\alpha$-hly genes $[16,17]$.

\section{Discussion}

We have recently determined the nucleotide sequence of the pEO5 $\alpha$-hly genes, which are commonly occurring in EPEC O26 strains from humans and animals [21]. Surprisingly, the $\alpha-h l y$ genes were $99.2 \%$ similar to that of pHly152 which originates from a murine $E$. coli strain. Moreover, plasmid encoded $\alpha$-hly genes from pHly 152 and $\mathrm{pEO} 5$ showed close similarity in the regulatory region upstream of $h l y C$ and thus differed from chromosomally inherited $\alpha$-hly determinants [21]. These findings may indicate that plasmid encoded $\alpha$-hemolysins have evolved from one source and separately from the chromosomal hemolysin operons. In order to explore this possibility we compared plasmid $\alpha-h l y$ from unrelated $E$. coli strains of human, mouse, canine and porcine origin for similarities the regulatory and structural genes and their adjacent sequences.

Plasmid encoded $\alpha$-hly determinants were found similar to each other in their genes $(h l y R, h l y C, h l y A$ and $h l y D)$ as well as in the adjacent sequences upstream and downstream of the $\alpha$-hly-operon. Plasmid encoded $h l y C$ and $h l y A$ genes showed typical alterations in the nucleotide and in the amino acid sequence compared to their chromosomally encoded homologues. Moreover, chromosomally encoded $\alpha$-hly genes were found different for the regions encompassing the $\alpha$-hly-operon. The finding that chromosomal $h l y C$ and $h l y A$ genes clustered separately and showed greater sequence diversity compared to the plasmid homologues suggests that plasmid $\alpha$-hly-genes have emerged more recently in E. coli and thus accumulated fewer changes compared to the chromosomal $\alpha$-hly genes.

It was previously suggested that $\alpha$-hly genes were acquired by strains of $E$. coli by horizontal gene transfer $[25,27,30]$. This hypothesis is supported by the location of chromosomally encoded hemolysin genes on pathogenicity islands $[13,14,16,17]$ and the flanking of plasmid encoded $\alpha$-hly genes by transposable elements [20,21]. A truncated IS911 element located downstream of the $h l y D$ gene was found in all $\alpha$-hly plasmids investigated in our study indicating that the plasmid encoded $\alpha-h l y$ determinants may have descended from a common progenitor [31]. We do not know much about the genetic similarity between the $\alpha$-hly plasmids investigated in this study, except that they show differences in size (48$157 \mathrm{~kb})$ and conjugation ability. Further investigation of plasmid backbone sequences could reveal if they have descended from a common progenitor. At present, we cannot exclude that the $\alpha$-hly determinant was transposed independently to different plasmids in E. coli.

Interestingly, plasmid pEO14 differed largely from all other $\alpha$-hly-plasmids investigated in this study. The nucleotide sequence analysis of its $\alpha$-hly genes and the 


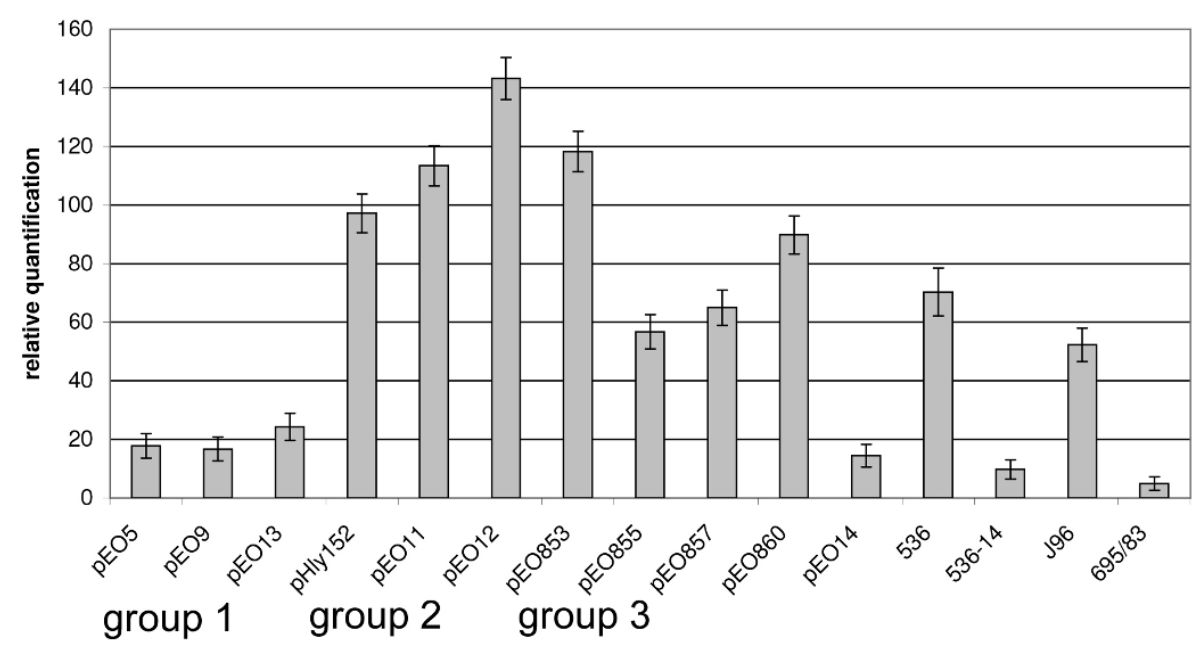

Figure 6 Relative quantification of the hlyA gene transcription in E. coli strains encoding plasmid and chromosomally inherited $\alpha$-hly determinants. Strains and plasmids as well as plasmid groups are listed in Table 1. Means and standard deviations from two separate experiments performed in duplicate are shown.

adjacent sequences revealed close similarity to chromosomal $\alpha$-hly genes. Because the $\alpha$ - $h l y$ genes present on plasmid pEO14 shows all features of chromosomal $\alpha-h l y$ operon it is likely that it was generated by recombination between a plasmid and chromosomal $\alpha$-hly loci in E. coli. A similar event might have been involved in generation of a truncated $\alpha$-hly segment in plasmid Vir68 that has been analyzed for its complete nucleotide sequence [GenBank CP001162].

The chromosomally located $\alpha$-hly genes of the $E$. cloacae strain KK6-16 showed similarities to E. coli plasmid encoded $\alpha$-hly determinants. The nucleotide sequence analysis of the $552 \mathrm{bp}$ upstream region of the $\alpha$-hly operon of KK6-16 was identical to that of the $\alpha$-hly plasmid pANN681 [GeneBank L01627] [32] and the "pHly152" region found in all $\alpha$-hly plasmids except pEO14 is present in KK6-16. Since $\alpha-h l y$ is not common in strains of Enterobacter species [26], it seems likely that strain KK6-16 acquired the $\alpha$-hly genes by conjugation from E. coli. Similar findings have been made for plasmids encoding antimicrobial resistance $[33,34]$. However, we have not investigated this possibility.

Interestingly, the $h l y C$ and $h l y \mathrm{~A}$ sequences of the KK6-16 showed characteristic features which made it difficult to assign its $\alpha$-hly determinant to the group of plasmid- or chromosomally inherited $\alpha$-hly genes (Figs. $4+5)$. It is possible that characteristic alterations found in the KK6-16 $\alpha$-hly sequence are due to E. cloacae as a different bacterial host species.

Multiple copies of IS1 and IS2 were frequently found in genetically unrelated strains of E. coli. IS1 and IS2 were found to be non-randomly scattered in the genomes of wild-type E. coli strains [35-37]. IS-elements are involved in chromosomal rearrangements, integration of F-plasmids and transposition of genes [38] and thus could have been involved in the generation of $E$. coli $\alpha-h l y$ plasmids. Activation of downstream genes by presence of IS 1 and IS 2 elements in E. coli has been reported [39] and this could explain the relatively high hlyA transcription rates in plasmids carrying IS2 or IS1 and IS2. However, we have not tested this possibility experimentally and other factors such as plasmid copy numbers and differences between the $E$. coli host strains could have an influence on the transcription rates.

$\alpha$-hemolysin plasmids are frequently found in STEC strains producing Stx2e, agents of edema disease in pigs [40], and in ETEC strains producing heat-stable enterotoxin causing diarrhea in dogs [10]. The $\alpha$-hly plasmid pEO5 is closely associated with EPEC O26 strains as diarrheal agents of human infants and calves [21,41]. In contrast, E. coli strains carrying chromosomal $\alpha$-hly are associated with UPEC which are characterized by other virulence attributes and serotypes than ETEC, EPEC and STEC strains $[13,14,16,17]$. The association of $\alpha$-hly plasmids with intestinal and of chromosomal $\alpha$-hly determinants with extraintestinal strains points to a separate evolution in these two major groups of pathogenic E. coli.

\section{Conclusion}

Our results indicate that the $\alpha$-hly genes present on plasmids in ETEC, STEC and EPEC strains have a common origin. The presence of IS-sequences flanking the plasmid $\alpha$-hly genes suggest that these were introduced in $E$. coli by horizontal gene transfer. Plasmids were shown to play a role in the spread of $\alpha$-hly determinant 
to Enterobacter cloacae. Chromosomally $\alpha$-hly genes present in UPEC are genetically more diverse and seem to have evolved separately from the plasmid $\alpha-h l y$ genes.

\section{Methods \\ Bacteria}

The bacterial strains used in this work are listed in Table 1. Strain C4115, the source of the plasmid pEO5, the $E$. coli strain 374 carrying the $\alpha$-hly-plasmid pHly152 as well as other EPEC O26 strains were described previously [21]. The relevant characteristics of strains with chromosomally located $\alpha$-hemolysin determinants are listed elsewhere $[10,18,19]$. The $\alpha$-hemolytic E. cloacae strain KK616 as well as the canine and porcine ETEC and STEC strains carrying $\alpha$-hly plasmids were described previously $[10,26,29,42]$. The EHEC-hemolysin plasmid pO157 carrying strain TPE1313 was used as negative control is described elsewhere [21]. Mating of bacteria with $E$. coli $\mathrm{K}-12$ recipient strains and isolation of $\alpha$-hemolytic transconjugants was performed as described by Burgos et al. 2009 [21]. Phenotypes corresponding to $E$. coli $\alpha$-hemolysin were analyzed on washed sheep blood agar [43].

\section{Isolation of DNA, RNA and CDNA}

Total DNA of bacteria was isolated as described [29]. Purified plasmid DNA of bacteria that was used for restriction digestion, DNA-hybridization, PCR and nucleotide sequencing was isolated with the large construct kit following the instructions of the producer (Qiagen, Hilden, Germany). Analysis of total plasmid profiles of $E$. coli strains was performed as described previously [44]. Total RNA was isolated from $20 \mathrm{ml}$ of exponentially growing aerated cultures $\left(3-5 \times 10^{8}\right.$ bacteria/ml) of bacteria in L-Broth with the RNeasy minikit (Qiagen). Isolation of RNA and preparation of cDNA was performed as described previously [29].

\section{DNA hybridization}

Southern blot hybridization of plasmid DNA and labeling of gene probes with Digoxigenin-11-dUTP was performed as described [21]. Dig-labeled molecular markers (Dig Roche) were used for size determination of hybridizing DNA fragments. For identification of $\alpha$-hly plasmids in Southern blotted gels a $666 \mathrm{bp}$ PCR product of the $\alpha-h l y A$ gene generated with primers $10 \mathrm{f} / \mathrm{r}$ (Table 2) was used as internal DNA probe for detection of $\alpha-h l y$ specific sequences [21]. Plasmids pHly152, pO157 and pEO5 served as reference plasmids for size determination of $\alpha$-hly plasmids [21] (Fig. 1).

\section{Nucleotide sequencing of $\alpha$-hemolysin and associated sequences}

Nucleotide sequence analysis of the $\alpha$-hly determinants and adjacent sequences was performed as described [21].
PCR products were purified and used for sequencing applying the dye terminator chemistry (PE Applied Biosystems, Darmstadt, Germany) and separated on an automated DNA sequencer (ABI PRISM ${ }^{\circledR} 3100$ Genetic Analyzer, Applied Biosystems, Foster City, CA). The sequences were analyzed using the Lasergene software (DNASTAR, Madison,WI) and Accelrys Gene v2.5 software.

\section{Development of specific PCRs for plasmid- and chromosomally inherited $\alpha$-hly determinants and their associated sequences}

Primer pairs specific for $\alpha$-hly-plasmid specific sequences $h l y R$ (primers $44 \mathrm{f} / \mathrm{r}$ ), the region between $h l y R$ and $h l y C$ (primers 1f/r, 32f/r), hlyA (111f/r and 113f/r) and $h l y D$ and downstream (99f/r) (Table 2) were developed with Accelrys software using the pEO5 sequence [GenBank FM180012]. Primer pairs for amplification of regions upstream of $h l y C$ in chromosomally inherited $\alpha-$ hemolysins were developed from sequences of PAI I $(81 \mathrm{f} / \mathrm{r})$ and PAI II (72f/r) from the uropathogenic E. coli strain 536 (Tables $1+2$ ). Primers $10 \mathrm{f} / \mathrm{r}$ served as positive control for general detection of plasmid and chromosomally inherited $\alpha$-hly determinants. Primers and PCR conditions are listed in Table 2. PCR reactions were performed as described previously [29].

\section{Transcriptional analysis of $\alpha-h l y A$ genes by qRT-PCR}

Quantitative real time reverse transcription PCR (qRTPCR) was performed with the Applied Biosystems 7500 real time PCR system (Applied Biosystems) with cDNA samples from bacteria (see above). Transcription rates of the $\alpha-h l y A$ gene were compared to those of the $i c d A$ housekeeping gene. Primers hlyA-f 5' ACCTTGTCAGGACGGCAGAT 3' and hlyA-r 5' CCGTGCCATTC TTTTCATCA 3 ' and the VIC labeled TaqMan MGB probe 5' ACTGGGAATTGAAGTCC 3' were used for amplification of the $\alpha-h l y A$ gene. The primers and the gene probe for detection of the icdA gene were described recently [29]. Real time PCR amplification were performed in an "icdA \& $\alpha$-hlyA" multiplex assay and were analyzed with the 7500 system SDS software version 1.4 as described [29].

\section{GenBank accession numbers}

The following nucleotide sequences derived from the $\alpha$ hemolysin producing strains and $\alpha$-hly plasmids from Table 1 were submitted to GenBank: strain 374 (pHly152) [GenBank FN678785]; 84-2195 (pEO9) [GenBank FM210248, FN673699, FN678787]; 84-3208 (pEO11) [GenBank FM210249, FN678787, FN673696]; CB853 (pEO853) [GenBank FM210347, FN678782, FN673701]; 84-R (pEO13) [GenBank FM210348, FN678786, FN673698]; 84-2573 (pEO12) [FM210349, 
FN678784, FN673703]; 84-2 S (pEO14) [GenBank FM210350, FN673697]; CB860 (pEO860) [GenBank FM210351, FN678780, FN673700]; CB855 (pEO855) [GenBank FN678788]; CB857 (pEO857) [GenBank (FN678781, FN673702] and strain KK6-16 [FM210352, FN673704].

\section{Acknowledgements}

Y. Burgos was partially supported from Brazil by "Fundação de Amparo à Pesquisa do Estado de São Paulo (FAPESP)", process of number 2006//538052. The authors are grateful to Eckhard Strauch (BfR, Berlin) for valuable discussions and suggestions and to Karin Pries for technical assistance.

\section{Authors' contributions}

LB took an integral part of project conception and both YB and LB in method development. YB took most part in the design and performance of the experimental procedures. Data analysis was performed by both researchers $L B$ and $Y B$ as well as interpretation of results and preparation of the manuscript. All authors have read and approved the final manuscript

Received: 30 March 2010 Accepted: 19 July 2010

Published: 19 July 2010

\section{References}

1. Welch RA: Pore-forming cytolysins of gram-negative bacteria. $\mathrm{Mol}$ Microbiol 1991, 5:521-528

2. Menestrina G, Moser C, Pellet S, Welch R: Pore-formation by Escherichia coli hemolysin (HlyA) and other members of the RTX toxins family. Toxicology 1994, 87:249-267.

3. Stanley P, Koronakis V, Hughes C: Acylation of Escherichia coli hemolysin: a unique protein lipidation mechanism underlying toxin function. Microbiol Mol Biol Rev 1998, 62:309-333.

4. Schmidt H, Kernbach C, Karch H: Analysis of the EHEC hly operon and its location in the physical map of the large plasmid of enterohaemorrhagic Escherichia coli 0157:h7. Microbiology 1996, 142(Pt 4):907-914.

5. Holland IB, Schmitt L, Young J: Type 1 protein secretion in bacteria, the ABC-transporter dependent pathway (review). Mol Membr Biol 2005, 22:29-39.

6. Schmidt $\mathrm{H}$, Karch $\mathrm{H}$, Beutin L: The large-sized plasmids of enterohemorrhagic Escherichia coli 0157 strains encode hemolysins which are presumably members of the E. coli alpha-hemolysin family. FEMS Microbiol Lett 1994, 117:189-196.

7. Brunder W, Schmidt H, Frosch M, Karch $H$ : The large plasmids of Shigatoxin-producing Escherichia coli (STEC) are highly variable genetic elements. Microbiology 1999, 145(Pt 5):1005-1014.

8. Johnson JR: Virulence factors in Escherichia coli urinary tract infection. Clin Microbiol Rev 1991, 4:80-128.

9. Bertschinger HU, Gyles CL: Oedema Disease of Pigs. Escherichia coli in domestic animals and humans Wallingford: CAB InternationalGyles CL 1994, 193-219.

10. Prada J, Baljer G, De Rycke J, Steinruck H, Zimmermann S, Stephan R, et al: Characteristics of alpha-hemolytic strains of Escherichia coli isolated from dogs with gastroenteritis. Vet Microbiol 1991, 29:59-73.

11. Hampson DJ: Postweaning Escherichia coli Diarrhoea in Pigs. Escherichia coli in Domestic animals and Humans Wallingford: CAB InternationalGyles CL 1994, 171-191.

12. Beutin L: Escherichia coli as a pathogen in dogs and cats. Vet Res 1999, 30:285-298.

13. Guyer DM, Kao JS, Mobley HL: Genomic analysis of a pathogenicity island in uropathogenic Escherichia coli CFT073: distribution of homologous sequences among isolates from patients with pyelonephritis, cystitis, and Catheter-associated bacteriuria and from fecal samples. Infect Immun 1998, 66:4411-4417.

14. Kao JS, Stucker DM, Warren JW, Mobley HL: Pathogenicity island sequences of pyelonephritogenic Escherichia coli CFT073 are associated with virulent uropathogenic strains. Infect Immun 1997, 65:2812-2820.
15. Dobrindt U, Janke B, Piechaczek K, Nagy G, Ziebuhr W, Fischer G, et al: Toxin genes on pathogenicity islands: impact for microbial evolution. Int J Med Microbiol 2000, 290:307-311.

16. Swenson DL, Bukanov NO, Berg DE, Welch RA: Two pathogenicity islands in uropathogenic Escherichia coli J96: cosmid cloning and sample sequencing. Infect Immun 1996, 64:3736-3743.

17. Dobrindt U, Blum-Oehler G, Nagy G, Schneider G, Johann A, Gottschalk G, et al: Genetic structure and distribution of four pathogenicity islands (PAI I(536) to PAI IV(536)) of uropathogenic Escherichia coli strain 536. Infect Immun 2002, 70:6365-6372.

18. Nagy G, Altenhoefer A, Knapp O, Maier E, Dobrindt U, Blum-Oehler G, et al: Both alpha-haemolysin determinants contribute to full virulence of uropathogenic Escherichia coli strain 536. Microbes Infect 2006, 8:2006-2012.

19. Prada J, Zimmermann S, Stephan R, Beutin L: Restriction fragment length polymorphisms associated with alpha-hemolysin determinants are correlating with the expression of alpha-hemolysin in strains of Escherichia coli. Zentralbl Bakteriol 1992, 276:152-164.

20. Knapp S, Then I, Wels W, Michel G, Tschape H, Hacker J, et al: Analysis of the flanking regions from different haemolysin determinants of Escherichia coli. Mol Gen Genet 1985, 200:385-392.

21. Burgos YK, Pries K, Pestana de Castro AF, Beutin L: Characterization of the alpha-haemolysin determinant from the human enteropathogenic Escherichia coli O26 plasmid pEO5. FEMS Microbiol Lett 2009, 292:194-202.

22. Wu XY, Chapman T, Trott DJ, Bettelheim K, Do TN, Driesen S, et al: Comparative analysis of virulence genes, genetic diversity, and phylogeny of commensal and enterotoxigenic Escherichia coli isolates from weaned pigs. Appl Environ Microbiol 2007, 73:83-91.

23. Grunig HM, Lebek G: Haemolytic activity and characteristics of plasmid and chromosomally borne hly genes isolated from E. coli of different origin. Zentralbl Bakteriol Mikrobiol Hyg [A] 1988, 267:485-494.

24. Hess J, Wels M, Vogel M, Goebel W: Nucleotide sequence of a plasmidencoded hemolysin determinant and its caomparison with a corresponding chromosomal hemolysin sequence. FEMS Microbiol Lett 1986, 34:1-11.

25. Strathdee CA, Lo RY: Extensive homology between the leukotoxin of Pasteurella haemolytica A1 and the alpha-hemolysin of Escherichia coli. Infect Immun 1987, 55:3233-3236.

26. Prada J, Beutin L: Detection of Escherichia coli alpha-haemolysin genes and their expression in a human faecal strain of Enterobacter cloacae. FEMS Microbiol Lett 1991, 63:111-114.

27. Koronakis V, Cross M, Senior B, Koronakis E, Hughes C: The secreted hemolysins of Proteus mirabilis, Proteus vulgaris, and Morganella morganii are genetically related to each other and to the alphahemolysin of Escherichia coli. J Bacteriol 1987, 169:1509-1515.

28. Vogel M, Hess J, Then I, Juarez A, Goebel W: Characterization of a sequence (hlyR) which enhances synthesis and secretion of hemolysin in Escherichia coli. Mol Gen Genet 1988, 212:76-84

29. Beutin L, Kruger U, Krause G, Miko A, Martin A, Strauch E: Evaluation of major types of Shiga toxin 2e producing Escherichia coli present in food, pigs and in the environment as potential pathogens for humans. Appl Environ Microbiol 2008.

30. Strathdee CA, Lo RY: Cloning, nucleotide sequence, and characterization of genes encoding the secretion function of the Pasteurella haemolytica leukotoxin determinant. J Bacteriol 1989, 171:916-928.

31. Gueguen E, Rousseau P, Duval-Valentin G, Chandler M: Truncated forms of IS911 transposase downregulate transposition. Mol Microbiol 2006, 62:1102-1116.

32. Frechon D, Le Cam E: Fur (ferric uptake regulation) protein interaction with target DNA: comparison of gel retardation, footprinting and electron microscopy analyses. Biochem Biophys Res Commun 1994, 201:346-355.

33. Khalaf NG, Eletreby MM, Hanson ND: Characterization of CTX-M ESBLs in Enterobacter cloacae, Escherichia coli and Klebsiella pneumoniae clinical isolates from Cairo, Egypt. BMC Infect Dis 2009, 9:84.

34. Galani I, Souli M, Koratzanis E, Koratzanis G, Chryssouli Z, Giamarellou H: Emerging bacterial pathogens: Escherichia coli, Enterobacter aerogenes and Proteus mirabilis clinical isolates harbouring the same transferable plasmid coding for metallo-beta-lactamase VIM-1 in Greece. J Antimicrob Chemother 2007, 59:578-579. 
35. Sawyer SA, Dykhuizen DE, Dubose RF, Green L, Mutangaduramhlanga T, Wolczyk DF, et al: Distribution and Abundance of Insertion Sequences Among Natural Isolates of Escherichia-Coli. Genetics 1987, 115:51-63.

36. Boyd EF, Hartl DL: Nonrandom location of IS1 elements in the genomes of natural isolates of Escherichia coli. Mol Biol Evol 1997, 14:725-732.

37. Mahillon J, Leonard C, Chandler M: IS elements as constituents of bacterial genomes. Res Microbiol 1999, 150:675-687.

38. Bachellier S, Gilson E, Hofnung M, Hill CW: Repeated Sequences. Escherichia coli and Salmonella Washington D.C.: ASM PressNeidhardt FC, Curtiss R III, Ingraham J, Lin ECC, Low KB, Megasanik WS, et al , 2 2010, 2:2012-2040.

39. Galas DJ, Chandler M: Bacterial Insertion Sequences. Mobile DNA Washington D.C.: ASMBerg DE, Howe MM 1989, 109-162.

40. Jorgensen ST, Poulsen AL: Antibiotic resistance and Hly plasmids in serotypes of Escherichia coli associated with porcine enteric disease. Antimicrob Agents Chemother 1976, 9:6-10.

41. Leomil L, Pestana de Castro AF, Krause G, Schmidt H, Beutin L: Characterization of two major groups of diarrheagenic Escherichia coli O26 strains which are globally spread in human patients and domestic animals of different species. FEMS Microbiol Lett 2005, 249:335-342

42. Han W, Liu B, Cao B, Beutin L, Kruger U, Liu H, et al: DNA Microarray-Based Identification of Serogroups and Virulence Gene Patterns of Escherichia coli Isolates Associated with Porcine Postweaning Diarrhea and Edema Disease. Appl Environ Microbiol 2007, 73:4082-4088.

43. Beutin L, Zimmermann S, Gleier K: Rapid detection and isolation of Shigalike toxin (verocytotoxin)-producing Escherichia coli by direct testing of individual enterohemolytic colonies from washed sheep blood agar plates in the VTEC-RPLA assay. I Clin Microbiol 1996, 34:2812-2814.

44. Kado Cl, Liu ST: Rapid procedure for detection and isolation of large and small plasmids. J Bacteriol 1981, 145:1365-1373.

45. Tajima F, Nei M: Estimation of evolutionary distance between nucleotide sequences. Mol Biol Evol 1984, 1:269-285.

46. Welch RA, Hull R, Falkow S: Molecular cloning and physical characterization of a chromosomal hemolysin from Escherichia coli. Infect Immun 1983, 42:178-186.

doi:10.1186/1471-2180-10-193

Cite this article as: Burgos and Beutin: Common origin of plasmid encoded alpha-hemolysin genes in Escherichia coli. BMC Microbiology 2010 10:193.

\section{Submit your next manuscript to BioMed Central and take full advantage of:}

- Convenient online submission

- Thorough peer review

- No space constraints or color figure charges

- Immediate publication on acceptance

- Inclusion in PubMed, CAS, Scopus and Google Scholar

- Research which is freely available for redistribution 